\title{
AN ANALYTICAL MODEL FOR PREDICTION OF CONTROLLED RELEASE FROM BULK BIODEGRADING POLYMER MICROSPHERES
}

\author{
FARZANE SIVANDZADE*
}

Department of Chemical and Petroleum Engineering, Sharif University of Technology, Tehran, Iran. Email: farzane_sivandzade@yahoo.com

Received: 21 October 2017, Revised and Accepted: 28 November 2017

\begin{abstract}
Objective: A convenient numerical model has been developed to predict release profile of different types of agents from bulk biodegrading polymer microspheres, including magnitude methods are less accurate than analytical methods. Usually, this study used analytical solutions for the model and compared the analytical results with numerical solution and experimental data.
\end{abstract}

Methods: The objective drug controlled release profiles were modeled based on a four-phase pattern. Then, a specific formulation was considered based on Fick's second law. After calculating various parameters, the equations were solved using an analytical method.

Results: Comparison results showed that analytical solution can reproduce experimental behavior of controlled release systems with a higher accuracy.

Conclusion: Although in previous work, drug release profile from a polymer matrix composed of poly lactic-co-glycolic acid was predicted using readily attainable parameters and representing tunable matrix properties by a numerical method, the proposed analytical method can give more accurate results compared to the numerical method.

Keywords: Analytical method, Biodegrading polymer, Microsphere, Controlled release, Model.

(C) 2018 The Authors. Published by Innovare Academic Sciences Pvt Ltd. This is an open access article under the CC BY license (http://creativecommons. org/licenses/by/4. 0/) DOI: http://dx.doi.org/10.22159/ajpcr.2018.v11i3.23219

\section{INTRODUCTION}

Nowadays, drug controlled release technology has expanded, noticeably and, polymer matrices have become the most commonly applicable controlled release delivery system [1]. In controlled treatments, a polymer matrix, containing a drug or a pharmaceutical agent, is placed in the body and the embedded drug releases and distributes through a diffusional process. After complete removal of the drug, the polymer matrix can be removed by surgery. Due to limitations and difficulties of surgery, a wide variety of biodegradable polymers with various geometries have been employed in the field of pharmaceutical sciences [2-4]. In this type of matrix system, the polymeric materials consist of monomers linked to each other through functional groups with instable functionality. The degradation of polymeric materials into oligomers and monomers occurs through either biological enzymes produced by surrounding tissues or non-enzymatic processes [1]. In the context of controlled release technology, wide applicability of polymer matrices causes the development of numerous unique therapeutics to improve patients' quality of life and satisfaction [5]. Since in vitro experiments need spending long time and high costs, it is attempted to predict controlled release of drugs from biodegradable polymer matrices using modeling techniques which satisfy the three following requirements [6]:

1. The model should be described according to available parameters;

2. The model must include all functions of the release process; and

3. The model must be applicable to a wide range of drugs.

In this respect, many studies have attempted to model biodegradation and controlled drug release profiles based on physical properties of the matrix, the embedded drug, and the employed polymer [7-10]. In one of the first models describing drug release from a biodegradable polymer matrix, Thombre et al. [11] used finite element mathematics to calculate drug release based on Fick's second law, and, thus, effective diffusivity proportional to the extent of polymer degradation was incorporated into the model. In 1989, Saltzman et al. [12] described agent diffusion through non-biodegradable, porous polymer matrices, accurately, using percolation theory, and in vitro studies. In their proposed model, Fick's second law containing an effective diffusivity factor was used. The effective diffusivity factor could vary with the type of their polymer matrix. Gopferich et al. [13] studied stochastic methods to describe time evolution of increased porosity of biodegradable systems and calculated release of water-soluble small molecules from polyanhydride disks, which were assumed to degrade through surface erosion. In a different approach, Batycky et al. [14] developed a model based on differential equations to describe burst-lag-burst type release from polyester microparticles. Later, Siepmann et al. [9] described the release of a low-molecular-weight agent from bulk eroding poly lacticco-glycolic acid (PLGA) microspheres. To improve the description of drug release, they added some equations governing porosity dependent diffusion of the agent and performed Monte Carlo simulations. Rothstein et al. [6] developed a simple mathematical model, which was solved using numerical method, to predict the release of different types of agents from bulk eroding polymer matrices without regression analysis. In their model, two correlations were developed by fitting the fundamental equations to the published controlled release data so that their predictions for several different biodegradable matrix systems used easily measurable or commonly known parameters. It was shown that the model predicts a wide range of therapeutically relevant release behaviors. They calculated a finite element solution for the given matrix geometry using some default solver settings. Due to high symmetry of spheres or high aspect ratio of thin films, the modeled matrix geometries were simplified to be one dimensional to decrease computation time. They validated their numerical solutions of the model by fitting to experimental data for varying molecular weight of release $\left(M W_{r}\right)$ and diffusivity of the agent through the porous matrix $(D)$.

Each of the mentioned models follow successful steps toward enabling rational design of biodegradable controlled release polymer matrices by solving the related equations through numerical methods. However, numerical methods are less accurate than analytical methods. In 
fact, the biggest advantage of analytical method is building a degree of confidence for both the developer and the user. Although the analytical method may appear time consuming, it results inexpensive, eliminates frustrating repetitions, and causes better time management in the end [15]. Therefore, the main objective of the present work is the prediction of drug controlled release from biodegrading polymer matrices using analytical methods and comparing the results with numerical solutions and experimental data. In this work, a new method has been used to describe the release of several water-soluble agents such as gentamicin, insulin, leuprorelin, melittin, betamethasone, metoclopramide, and ethacrynic acid, discretely encapsulated in bulk biodegrading polymer matrices that dissolve rapidly, relative to the time scale of release. In this model, PLGA polymer has been used due to the fact that lactic acid and glycolic acid monomers, that would be produced during PLGA decomposition, are not harmful to body and are quite biocompatible. Moreover, PLGA is a time-dependent watersoluble polymer. In addition to the fundamental equations required for modeling agent release, the adopted model of this study includes two correlation functions that enable predictions with knowledge of five commonly known or easily measurable parameters. These parameters are microsphere radius $R_{p^{\prime}}$ occlusion radius $R_{o c c^{\prime}}$ polymer degradation rate constant $k C_{w^{\prime}}$ initial molecular weight of the polymer $M W_{g^{\prime}}$ and molecular weight of the releasing agent $M W_{A^{*}}$

\section{METHODS}

\section{Modeling}

Based on former studies on bulk biodegrading polymer matrices, drug controlled release profile can follow a four-phase pattern consisted of an initial burst phase, a lag phase, a secondary burst phase, and a final release phase. To model this pattern, first, an initially uniform matrix composed of a biodegradable polymer, i.e., PLGA, with randomly distributed and entrapped releasing agent with initial concentration of the agent in the polymer matrix $\left(C_{A 0}\right)$, which is loaded below its percolation threshold was considered [6].
To prepare drug containing polymer matrices, the agents, i.e. the embedded drugs, are usually encapsulated as a solution in occlusions so that they are dissolved in a solvent and then encapsulated in small spherical occlusions with radius $R_{\text {occ }}$. Then, the spherical occlusions are encapsulated within a polymer matrix, uniformly [3]. When the polymer matrix is implanted in body, at time zero, an aqueous reservoir begins to hydrate the matrix and an eroding reaction happens, quickly $[10,16]$. As the matrix hydrates, the encapsulated agent molecules adjacent to the matrix surface, with a direct pathway to egress, diffuse into the reservoir, rapidly, due to lack of any polymer on their pathways)the initial burst, phase 1 of the release pattern in Fig. 1). Relative size of the occlusion $\left(R_{\text {occ }}\right)$ occupied by the encapsulated agent is proportional to the magnitude of the initial burst phase, as illustrated in Fig. 2 [6]. As the initial burst release commences, polymer biodegradation begins and increases chain mobility, and it effectively leads to the formation of pores in the polymer matrix [17] (phase 2 of Fig. 1). In this model, a pore is defined as a region of polymer matrix with an average molecular weight low enough to allow release of the encapsulated agent. Further, the molecular weight of each encapsulated agent type associated with release may vary, and it might lead to a size-dependent restriction for agent egression. When pores form, the embedded agent diffuses gradually and its diffusion continues while enhancing number and diameter of the pores (the secondary burst; phase 3 of Fig. 1). Finally, cumulative growth and coalescence of the pores lead agents to diffuse toward the surface of the polymer matrix (phase 4 of Fig. 1) [18].

\section{Formulations}

Concentration of the agent in the polymer matrix $\left(C_{A}\right)$ within a matrix can be calculated from Fick's second law (Eq. 1) at any instant of time $(t)$ or any point of space $(r)$. When this equation is used, the agent is not generated or consumed in any reactions during its diffusion through the polymer matrix $[8,9]$

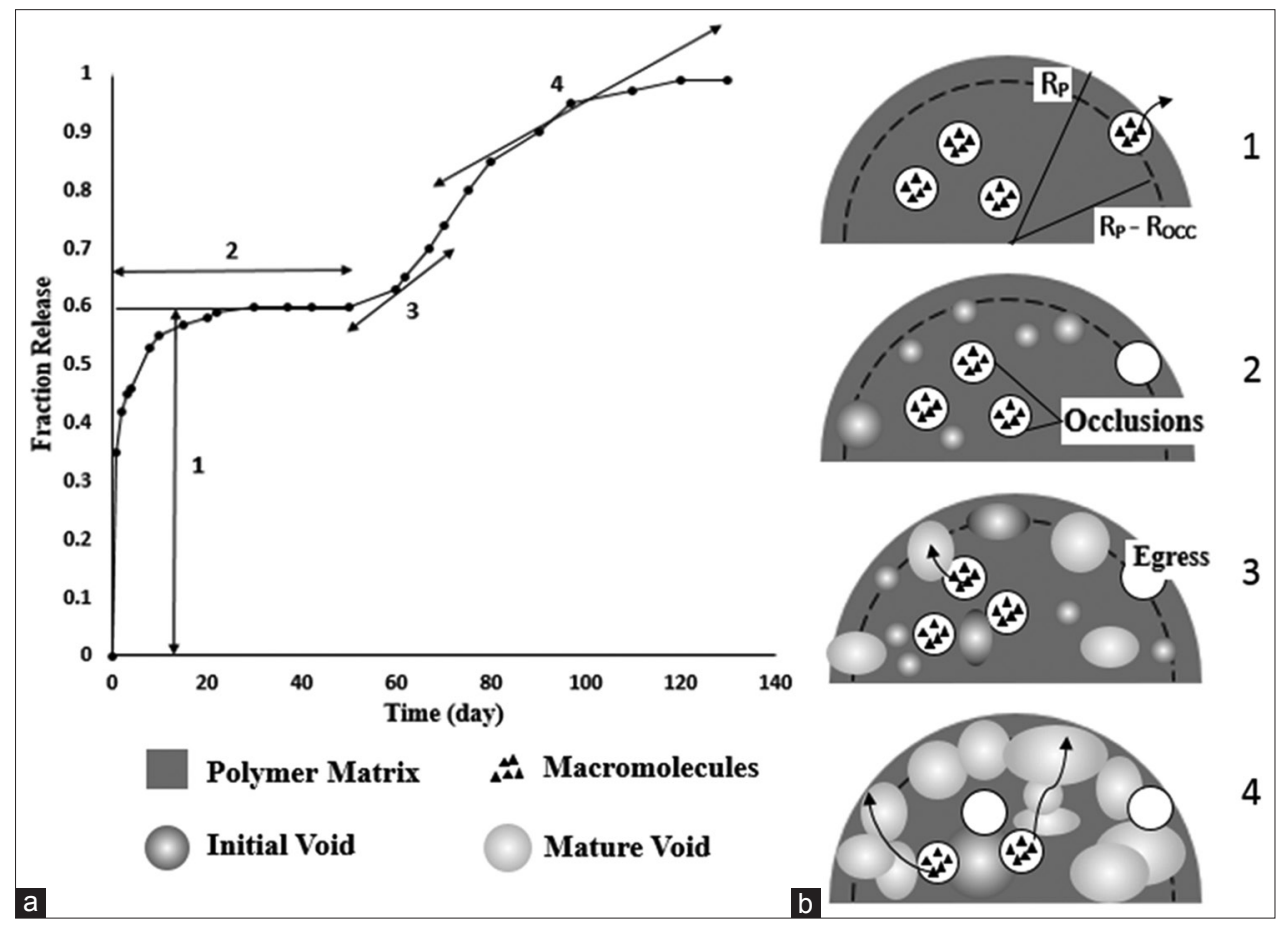

Fig. 1: Schematic depiction of the four-phase release pattern considered in the model. (a) Cross-section diagrams depicting the four phases of release for a double emulsion microparticle with an agent encapsulated in its occlusions, heterogeneously. (b) Release profile for a macromolecular drug encapsulated in a biodegradable polymer matrix with four phases of release. The numbers associated with each cross-section diagram indicate which phase of the release profile is illustrated. These phases are (1) the initial burst, (2) the lag phase, (3) the secondary burst, and (4) the final release [6] 


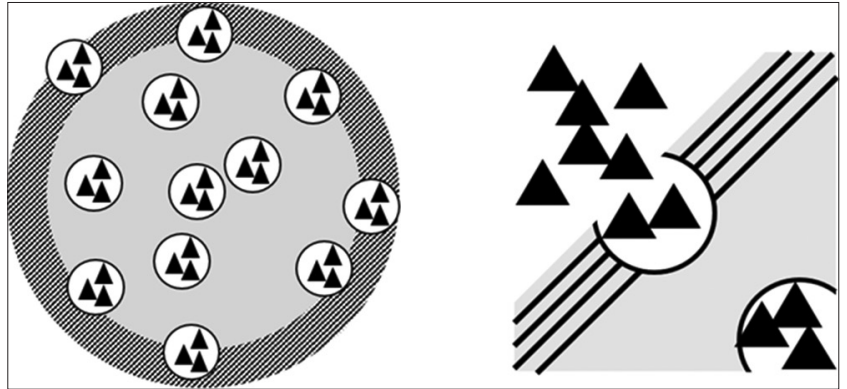

Fig. 2: Schematic presentation of the initial burst phase related to occlusion size. The double emulsion particle contains large occlusions filled with the drug solution

$\frac{\partial \mathrm{C}_{\mathrm{A}}}{\partial \mathrm{t}}=\frac{1}{\mathrm{r}^{2}} \frac{\partial}{\partial \mathrm{r}}\left(\mathrm{D}_{\mathrm{eff}} \mathrm{r}^{2} \frac{\partial \mathrm{C}_{\mathrm{A}}}{\partial \mathrm{r}}\right)$

In Eq. $1, D_{\text {eff }}$ is an effective diffusivity term and a boundary exists at a depth of $R_{\text {occ }}$ from the matrix surface $\left(r=R_{p}-R_{\text {occ }}\right)$ where continuity conditions are defined. In the subdomain of $R_{p}$ to $R_{p}-R_{o c c^{\prime}}$ the agent is subjected to the initial release phase so that $D_{\text {eff }}$ is simply a constant diffusivity $(D)$ which reflects the movement of the agent through the hydrated occlusions abutting the matrix surface. In the subdomain of 0 to $R_{p}-R_{o c,}$ the agent is subjected to molecular weight consideration and pore-dependent release. Therefore, the effects of these parameters on $D_{\text {eff }}$ should be concerned since $D_{\text {eff }}$ is related to porosity and porosity is a function of time for systems such as microspheres or sections in a thin film degrading heterogeneously. In this way, $D_{\text {eff }}=D \varepsilon(t)$, where $D$ is diffusivity of the agent through the porous matrix and $\varepsilon$ is timedependent matrix porosity. At the central point, line, or plane of the matrix $(r=0)$, symmetry conditions are defined $\left(d C_{A} / d r=0\right)$. At the matrix surface $\left(r=R_{p}\right)$, perfect sink conditions are specified $\left(C_{A}=0\right)$.

Assuming that polymer's degradation rate is normally distributed, induction time of pore formation can be concerned to follow a normal distribution [6]. Moreover, since pore formation is cumulative, timedependent matrix porosity $(\varepsilon(t))$ can be described with a cumulative normal distribution function (Eq. 2):

$\varepsilon(t)=\frac{1}{2}\left[\operatorname{erf}\left(\frac{t-\bar{\tau}}{\sqrt{2 \sigma^{2}}}\right)+1\right]$

In this equation, $t$ is any insant of time, $\bar{\tau}$ is the mean time for pore formation, and $\sigma^{2}$ is the variance of time required to form pores.

\section{Implementation}

Calculating $k C_{w}$ : Pseudo-first order degradation rate equations can be expressed as $\mathrm{MW}=\mathrm{MW}_{0} \mathrm{e}^{-\mathrm{kc}_{\mathrm{w}} \mathrm{t}}$. Consequently, polymer degradation rate constant $\left(k C_{w}\right)$ can be obtained from the slope of ln $M W$ versus $T$ curve, and $M W_{0}$ can be found from the intercept while $M W$ values measured at different time intervals can be obtained by averaging them from several valid sources [19-21].

Calculating $\bar{\tau}$ : The mean time for the formation of pores $(\bar{\tau})$ can be calculated through Eqs. 3 and 4:

$$
\begin{aligned}
& r A=-\frac{d M W_{r}}{d t}=k C_{w} M W_{r} \\
& \bar{\tau}=\frac{-1}{\mathrm{kC}_{\mathrm{w}}} \ln \left(\frac{\mathrm{MW}_{\mathrm{r}}}{\mathrm{MW}_{0}}\right)
\end{aligned}
$$

Where, $k C_{w}$ is the polymer degradation rate constant for the given polymer type, $M W_{o}$ is the initial molecular weight of the polymer, and $M W_{r}$ is the average polymer molecular weight in a differential volume of matrix allowing diffusion of the encapsulated agent. For blended polymer matrices, the value of $\bar{\tau}$ can be calculated by averaging the results obtained from Eq. 4 for each component. The matrix's molecular weight on release $\left(M W_{r}\right)$ specifies how far degradation is required before releasing the agent and how far it would vary depending on size of the encapsulated agent. Macromolecules or larger agents can only diffuse through a section of matrix that is almost entirely free of insoluble polymer chains. Smaller agents can egress through more intact sections of the polymer matrix (higher $M W_{r}$ ) and demand for less free space for passing.

Calculating $\sigma$ : Variance of time required to form pores $(\sigma)$ can be obtained according to Eq. 5 using mean time of pore formation $(\bar{\tau})$ and time for pore formation $(\tau)$. $\bar{\tau}$ can be calculated using $k C_{w}(n)$ values and a distribution of induction times $(t(n))$ based on Eq. 4:

$(\sigma)^{2}=\frac{1}{n-1} \sum_{i=0}^{n}(\tau-\bar{\tau})^{2}$

Calculating $M W_{r}$ and $D$ : Due to difficulty of measuring the values of agent diffusivity through the porous matrix $(D)$ and $M W_{r}$, these parameters should be calculated by linking them to several easily measurable variables. To calculate $M W_{r}$, the experimental values for a wide range of agents with different molecular weights have been presented by Rothstein et al. [6].

Several tests have been performed to show the influence of diameter on agent diffusion. Siepmann et al. [17] studied about the importance of autocatalysis in PLGA-based microparticles used as controlled drug delivery systems and confirmed that PLGA degrades into shorter chain alcohols and acids on contact with biological fluids. Accumulation of acids can lead to a significant drop in pH of PLGA microenvironment and accelerates subsequent polymer degradation. In other hand, the produced acid tends to leave the polymer by diffusion. However, as the mechanism of acid production is much faster than its diffusion toward outside of the polymer, $\mathrm{pH}$ is lower inside the polymer $[8,17]$. Therefore, the larger size of polymer creates a longer direction path for the produced acid; pH elevation occurs later, accumulation of acid in the polymer increases and as aforementioned, increased acidity leads to an increase in polymer decomposition rate and ultimately diffusivity of the agent. Since calculating diffusivity for each sample is difficult, fitting diffusivity versus diameter for the previous samples presents the relationship between them. Therefore, diffusivity was calculated through a regression analysis based on the power expression of $y=a X^{b}$ $\left(X=R_{p}\right.$ and $\left.y=D\right)$. According to experimental data [6] $a=2.071 \times 10^{-19}$ and $b=2.275$, which accurately corresponds to PLGA and can be extended to other polymers.

\section{Solutions}

Part A: Initial burst

Fick's second law and boundary conditions for the initial burst phase are:

$$
\begin{aligned}
& \frac{1}{\mathrm{r}^{2}} \frac{\partial}{\partial \mathrm{r}}\left(\mathrm{r}^{2} \frac{\partial \mathrm{C}_{\mathrm{A}}}{\partial \mathrm{r}}\right)=\frac{1}{\mathrm{D}} \frac{\partial \mathrm{C}_{\mathrm{A}}}{\partial \mathrm{t}} \\
& \text { Boundary.conditions: } \begin{cases}\frac{\partial C_{A}}{\partial r}=0 & r=0, t>0 \\
C A=0 & r=R_{p} \quad t>0\end{cases} \\
& \text { Initial condition } \mathrm{C}_{\mathrm{A}}=\mathrm{C}_{0} \quad \mathrm{t}=0
\end{aligned}
$$


Where, $C_{N}, D, r, t$, and $R_{p}$ are, respectively, agent's concentration, diffusivity of the agent through the porous matrix, any point of space, any instant of time, and microsphere radius, respectively. In 1975, Crank [22] solved this equation and found the final result as:

$\frac{m_{t 1}}{m_{\infty 1}}=1-\sum_{n=1}^{\infty} \frac{6}{n^{2} \pi^{2}} \exp \left(-\frac{D t}{R_{p}^{2}} n^{2} \pi^{2}\right)$

Where, $m_{t 1}$ is the magnitude of the available agent at any instant of time during the initial burst and $m_{\infty 1}$ is the magnitude of the available agent during the initial burst phase. It should be noted that total magnitude of the agent $\left(m_{\infty}\right)$, which can be removed, is equal to $m_{\infty 11}$ in $R_{p}-R_{o c c}$ distance, during the initial burst phase. The ratio $(\varphi)$ of this magnitude $\left(m_{\infty 1}\right)$ to total magnitude of the agent $\left(m_{\infty}\right)$, which represents the ratio of the first layer of matrix volume to total volume of the matrix, can be calculated as follows:

$\varphi=\frac{m_{\infty 1}}{m_{\infty}}=1-\frac{\left(R_{p}-R_{o c c}\right)^{3}}{R_{p}^{3}}$

Where, $R_{p}$ and $R_{\text {occ }}$ are microsphere radius and occlusion radius, respectively.

Part B: Lag phase (no mass transfer)

The pores are forming and the agent has been removed from the surface.

Part C: Secondary burst

In this phase, drug molecules pass out through the created pores and the pores become bigger gradually and, thus, diffusion increases. The relationship between effective diffusivity $\left(D_{e f f}\right)$ and matrix porosity $(\varepsilon)$ is defined to be:

$D_{\text {eff }}=D \varepsilon(t)$

Where, $D$ is diffusivity of the agent through the porous matrix.

Fick's second law and boundary conditions for the secondary burst phase are (diffusivity is time-dependent):

$$
\begin{aligned}
& \int \frac{1}{\mathrm{r}^{2}} \frac{\partial}{\partial \mathrm{r}}\left(\mathrm{r}^{2} \frac{\partial \mathrm{C}_{\mathrm{A}}}{\partial \mathrm{r}}\right)=\frac{1}{\mathrm{D}_{\mathrm{eff}}(t)} \frac{\partial \mathrm{C}_{\mathrm{A}}}{\partial \mathrm{t}} \\
& \text { Boundary.conditions: }\left\{\begin{array}{lll}
\frac{\partial \mathrm{C}_{\mathrm{A}}}{\partial \mathrm{r}}=0 & \mathrm{r}=0, \mathrm{t}>0 \\
\mathrm{CA}=0 & \mathrm{r}=\mathrm{R}_{\mathrm{p}} \quad \mathrm{t}>0
\end{array}\right. \\
& \text { Initial condition } \mathrm{C}_{\mathrm{A}}=\mathrm{C}_{0} \quad \mathrm{t}=0
\end{aligned}
$$

Where, $C_{A^{\prime}} D_{\text {eff }} r, t$, and $R_{p}$ are agent concentration, effective diffusivity, any point of space, any instant of time, and microsphere radius, respectively. To solve this equation, the variable should be changed:

$$
T=\int D_{\text {eff }}(t) d t
$$

The equation can then be solved by variable separation and the final result would be:

$$
\frac{m_{t 2}}{m_{\infty 2}}=1-\sum_{n=1}^{\infty} \frac{6}{n^{2} \pi^{2}} \exp \left(-\frac{T}{R_{p}^{2}} n^{2} \pi^{2}\right)
$$

Where, $m_{t 2}$ is the magnitude of the available agent at any instant of time during the secondary burst phase and $m_{\infty 2}$ is the magnitude of the available agent during the secondary burst phase. Integral $T$ was solved by Mathematica software (Mathematica 8.0.0 GNU/Linux frontend), and the following expression was achieved:

$$
\begin{aligned}
& T=\frac{1}{2} D\left(\frac{b \exp \left(-\left(\frac{t-\tau}{b}\right)^{2}\right)}{\sqrt{\pi}}+(t-\tau) \operatorname{erf}\left(\frac{t-\tau}{b}\right)+t\right) \\
& b=\sqrt{2 \sigma^{2}}
\end{aligned}
$$

Where, $D, t, \tau$, and $\sigma$ are diffusivity of the agent through the porous matrix, any instant of time, time for pore formation, and variance in time required to form pores, respectively. Total diffusion was obtained to be:

$$
\frac{\mathrm{m}_{\mathrm{t}}}{\mathrm{m}_{\infty}}=\varphi \frac{\mathrm{m}_{\mathrm{t} 1}}{\mathrm{~m}_{\infty 1}}+(1-\varphi) \frac{\mathrm{m}_{\mathrm{t} 2}}{\mathrm{~m}_{\infty 2}}
$$

Where, $m_{t 1}$ and $m_{t 2}$ are magnitudes of the available agent at any instant of time during the initial and secondary burst phases, respectively, $m_{\infty 1}$ and $m_{\infty 2}$ are magnitudes of the available agent during the initial and secondary burst phases, respectively, $m_{t}$ is total magnitude of the agent at any instant of time, $m_{\infty}$ is total magnitude of the agent, and $\varphi$ is the ratio of the first layer of matrix volume to total volume of the matrix.

\section{RESULTS AND DISCUSSION}

The results of analytical solutions are illustrated in Figs. 3 and 4. For validation of the analytical results of the model, they are fitted to experimental and numerical data using Matlab software (MATLAB R2013a) for varying $M W_{r}$ and $D$ values (Figs. 3 and 4). As illustrated in Figs. 3 and 4, analytical data are close to the experimental data, while the numerical solution of Rothstein et al. [6] has given results which are not close enough to the experimental data.

To prompt development of biodegrading matrices for controlled release therapeutics, many models have been developed. In general, these models describe the release of specific types of agents, such as small molecules or proteins $[7,9,10]$, and require parameters that can be only obtained by fitting controlled release data or observing controlled release experiments [9]. To eliminate the need of exploratory in vitro experiments and investigate drug dosing schedules supplied by potential controlled release therapeutics, a model must be able to predict a broad range of release behaviors from tunable matrices for a wide array of agents, without regression analysis. Hence, in the present work, we developed a new method to calculate the magnitude of the initial burst release and duration of the subsequent lag phase to permit these features to be predicted with commonly known parameters regardless of the encapsulated agent type. We solved this model using the parameters obtained by Rothstein et al. [6] to compare the results of both numerical and analytical methods.

To solve the fundamental equations using the developed analytical method, accurate values for $D$ and $M W_{,}, R_{o c c}, \bar{\tau}, k C_{w^{\prime}}$ and that have been obtained and used by Rothstein et al. [6] are required. Two partial differentiation equations with different diffusivities were solved to find accurate data close to the related experimental data. First, the equations were solved in terms of diffusivity given by Rothstein et al. [6], which resulted in analytical data that are different from the experimental values. To solve this problem, drug release profiles were obtained with different diffusivities by optimization of the diffusivity values. So that, the analytical data were modified to match the experimental data and magnitude of 


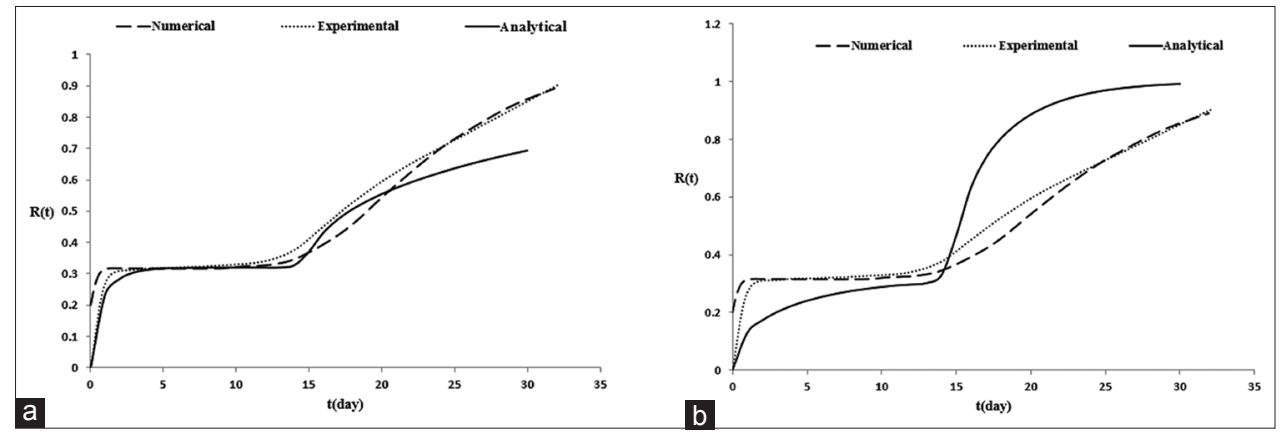

Fig. 3: Release profile of a peptide from poly lactic-co-glycolic acid (PLGA) microspheres. $M W_{r}$ for melittin $\left(M W_{A}=2.86 \mathrm{kDa}\right)$ was calculated to be $4.68 \mathrm{kDa}$. (a) Analytical solution according to the diffusivity value calculated by numerical method $\left(D=6.34 \times 10^{-18}[6]\right)$ for the $9.3 \mathrm{kDa}$ 75:25 PLGA microsphere, $R_{p}=4.5 \mu \mathrm{m}$ and $R_{o c c}=0.54 \mu \mathrm{m}$. (b) Analytical solution using optimal diffusivity value $\left(D=2 \times 10^{-17}\right.$ in the initial burst and $D=6 \times 10^{-19}$ in the secondary burst [6])

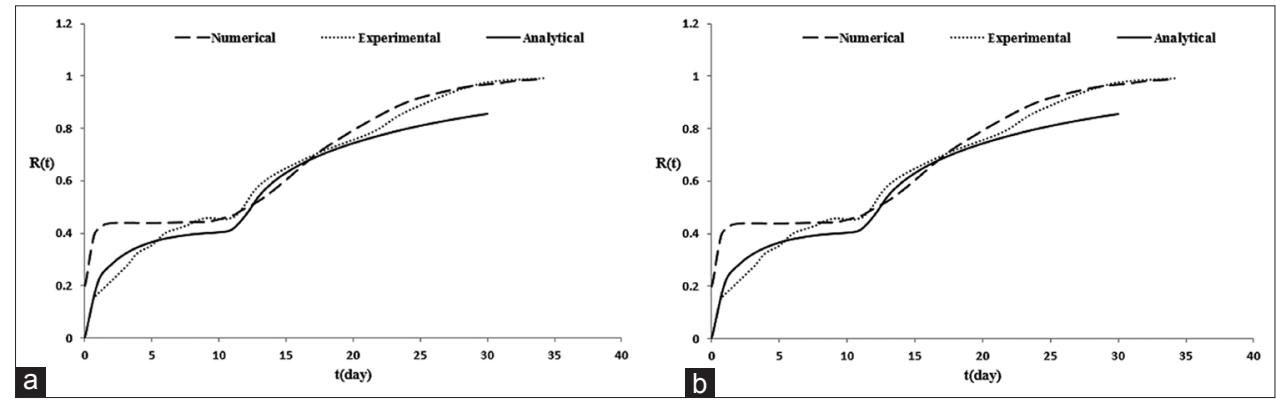

Fig. 4: Release profile of bovine serum albumin from polyanhydride composed of 20:80 CPH (1,6-bis-p-carboxyphenoxy hexane): SA (sebacic anhydride) polyanhydride $\left(M W_{0}=18 \mathrm{kDa}, R_{p}=10 \mu \mathrm{m}, R_{o c c}=1.54 \mu \mathrm{m}\right.$, and $M W_{r}$ are predicted to be $0.94 \mathrm{kDa}$ ). (a) Analytical solution according to the diffusivity value used in the numerical method $\left(D=6.34 \times 10^{-18}[6]\right)$ and (b) Analytical solution using optimal diffusivity value $\left(D=2 \times 10^{-17}\right.$ in the initial burst and $D=6 \times 10^{-19}$ in the secondary burst [6])

appropriate diffusivity was obtained. Although the optimum diffusivity value is slightly different from the diffusivity value given by Rothstein et al. [6], it affects curve fitting, noticeably. In fact, in analytical solution, various diffusivity values were assumed in modeling the initial and secondary burst phases since diffusivity in the secondary burst phase is more than that in the initial burst phase. This difference in diffusivities of the two burst phases is due to a sudden release caused by direct pathways of regression. However, Rothstein et al. [6] have assumed the same magnitude for both diffusivities. Since the diffusivity equation in terms of radius (in the work of Rothstein et al. [6]) has been obtained using data extracted from different references, i.e., the data might be obtained using different techniques, it is not quite accurate to relate these data in the same graph and fit them based on the same equation. Overall, according to the obtained analytical results, the model proposed by Rothstein et al., is an appropriate model for prediction of release profile of different types of agents from bulk biodegrading polymer microsphere. However, as the numerical method of Rothstein et al. [6] can only provide approximate solutions, the analytical method is revealed to be more useful.

\section{CONCLUSION}

In this study, a simple model that can predict controlled drug release successfully is demonstrated for an extremely wide array of agents encapsulated in bulk biodegrading polymer matrices. Although Rothstein et al. [6] have predicted drug release profile from a polymer matrix composed of PLGA using readily attainable parameters and representing tunable matrix properties by numerical method, this study solves the model by an analytical method than can give more accurate results compared to their numerical method.

\section{AUTHOR CONTRIBUTION}

Maryam Soleymani contributed in modelling, computational framework and the data analyzation.

\section{CONFLICT OF INTERERST}

No potential conflict of interest.

\section{REFERENCES}

1. Arafat M. Approaches to achieve an oral controlled release drug delivery system using polymers: A recent review. Int J Pharm Pharm Sci 2015;7:16-21.

2. Freiberg S, Zhu XX. Polymer microspheres for controlled drug release. Int J Pharm 2004;282:1-8

3. Jain RA. The manufacturing techniques of various drug loaded biodegradable poly(lactide-co-glycolide) (PLGA) devices. Biomaterials 2000;21:2475-90.

4. Jiang W, Gupta RK, Deshpande MC, Schwendeman SP. Biodegradable poly(lactic-co-glycolic acid) microparticles for injectable delivery of vaccine antigens. Adv Drug Deliv Rev 2005;57:391-410.

5. Rothstein SN, Federspiel WJ, Little SR. A unified mathematical model for the prediction of controlled release from surface and bulk eroding polymer matrices. Biomaterials 2009;30:1657-64.

6. Rothstein SN, Federspiel WJ, Little SR. A simple model framework for the prediction of controlled release from bulk eroding polymer matrices. J Mater Chem 2008;18:1873-80.

7. Siepmann J, Göpferich A. Mathematical modeling of bioerodible, polymeric drug delivery systems. Adv Drug Deliv Rev 2001;48:229-47.

8. Arifin DY, Lee LY, Wang CH. Mathematical modeling and simulation of drug release from microspheres: Implications to drug delivery systems. Adv Drug Deliv Rev 2006;58:1274-325.

9. Siepmann J, Faisant N, Benoit JP. A new mathematical model quantifying drug release from bioerodible microparticles using monte carlo simulations. Pharm Res 2002;19:1885-93.

10. Raman C, Berkland C, Kim KK, Pack DW. Modeling small-molecule release from PLG microspheres: Effects of polymer degradation and nonuniform drug distribution. J Controll Release 2005;103:149-58.

11. Thombre A, Himmelstein K. A simultaneous transport-reaction model for controlled drug delivery from catalyzed bioerodible polymer 
matrices. AIChE J 1985;31:759-66.

12. Saltzman WM, Langer R. Transport rates of proteins in porous materials with known microgeometry. Biophys J 1989;55:163-71.

13. Göpferich A, Langer R. Modeling of polymer erosion in three dimensions: Rotationally symmetric devices. AIChE J 1995;41:2292-9.

14. Batycky RP, Hanes J, Langer R, Edwards DA. A theoretical model of erosion and macromolecular drug release from biodegrading microspheres. J Pharm Sci 1997;86:1464-77.

15. Ravichandran V, Shalini S, Sundram K, Harish R. Validation of analytical methods-strategies and importance. Int J Pharm Pharm Sci 2010;2:18-22

16. Von Burkersroda F, Schedl L, Göpferich A. Why degradable polymers undergo surface erosion or bulk erosion. Biomaterials 2002;23:4221-31.

17. Siepmann J, Elkharraz K, Siepmann F, Klose D. How autocatalysis accelerates drug release from PLGA-based microparticles: A quantitative treatment. Biomacromolecules 2005;6:2312-9.
18. Fredenberg S, Reslow M, Axelsson A. Measurement of protein diffusion through poly(D,L-lactide-co-glycolide). Pharm Dev Technol 2005;10:299-307.

19. Panyam J, Dali MM, Sahoo SK, Ma W, Chakravarthi SS, Amidon GL, et al. Polymer degradation and in vitro release of a model protein from poly (D, L-lactide-co-glycolide) nano-and microparticles. J Controll Release 2003;92:173-87.

20. Göpferich A, Teßmar J. Polyanhydride degradation and erosion. Adv Drug Delivery Rev 2002;54:911-31.

21. Grayson AC, Voskerician G, Lynn A, Anderson JM, Cima MJ, Langer $\mathrm{R}$, et al. Differential degradation rates in vivo and in vitro of biocompatible poly(lactic acid) and poly(glycolic acid) homo- and co-polymers for a polymeric drug-delivery microchip. J Biomater Sci Polym Ed 2004;15:1281-304.

22. Crank J. The Mathematics of Diffusion. $2^{\text {nd }}$ ed. United states: Clarendon Press; 1975. 